OAI-PMH: http://www.indteca.com/ojs/index.php/Revista Scientific/oai

Artículo Original / Original Article

\title{
Patios Productivos como Estrategia para la Consolidación de la Educación Ambiental
}

\author{
Autora: Bárbara Ysabel Díaz Vásquez \\ Universidad Pedagógica Experimental Libertador, UPEL \\ marlenism3@gmail.com \\ Barinas, Venezuela
}

\begin{abstract}
Resumen
El estudio centró el objetivo general en proponer patios productivos como estrategia para la consolidación de la educación ambiental en los docentes de la Escuela Básica "Francisco Rivas", ubicada en el municipio Barinas, estado Barinas. La investigación estuvo enmarcada en el enfoque cuantitativo, insertado en la modalidad de Proyecto Factible y apoyado en un estudio campo de carácter descriptivo. La población y muestra estuvo constituida por sesenta y cinco (65) educadores de la institución antes referida. De allí, se tomó en consideración la totalidad de la misma para la muestra. En cuanto, a la recolección de la información se utilizó una encuesta tipo cuestionario tipo Likert con cinco (05) alternativas de respuestas: Siempre, Casi Siempre, Algunas Veces, Casi Nunca y Nunca. Por consiguiente, el mismo fue validado por jueces o expertos aplicando el coeficiente de consistencia interna de Cronbach (alfa). El estudio concluye: Los profesionales de la docencia necesitan la utilización del desarrollo sustentable en la consolidación de la educación ambiental; así como los docentes carecen de la utilización del desarrollo endógeno en la promoción de los patios productivos como estrategia en la institución. También, tienen ausencia de emplear los valores ambientales para impulsar los patios productivos en la institución y comunidad. Los educadores con poca frecuencia mantienen un comportamiento con una actitud positiva hacia la consolidación de los patios productivos en la escuela.
\end{abstract} ambiente.

Palabras clave: seguridad alimentaria; educación ambiental; medio 


\title{
Productive Patios as a Strategy for the Consolidation of Environmental Education
}

\begin{abstract}
The study focused on the general objective of proposing productive playgrounds as a strategy for the consolidation of environmental education in the teachers of the "Francisco Rivas" Basic School, located in the Barinas municipality, Barinas state. The research was framed in the quantitative approach, inserted in the Feasible Project modality and supported by a descriptive field study. The population and sample consisted of sixty-five (65) educators from the aforementioned institution. From there, all of it was taken into consideration for the sample. As for the collection of the information, a Likert-type questionnaire with five (05) alternative answers was used: Always, Almost Always, Sometimes, Almost Never and Never. Therefore, it was validated by judges or experts applying Cronbach's internal consistency coefficient (alpha). The study concludes: Teaching professionals need the use of sustainable development in the consolidation of environmental education; as well as teachers lack the use of endogenous development in the promotion of productive playgrounds as a strategy in the institution. Also, they have no use of environmental values to promote the productive courtyards in the institution and community. Educators infrequently maintain a positive attitude towards the consolidation of productive playgrounds at school.
\end{abstract}

Keywords: food security; environmental education; environment.

Date Received: 15-05-2018

Date Acceptance: 02-07-2018 


\section{Introducción}

La educación ambiental es un área que se encuentra en constante construcción permanente, sus inicios empiezan con la génesis de la humanidad transcurridos en varias épocas $y$, adquiriendo mucho auge con el conocimiento de la crisis ambiental en los tiempos actuales. Es así como, el último siglo, el contexto ambiental interrumpe en los aspectos sociales, políticos, educativos, culturales y tecnológicos; con un recorrido influenciado por conceptos actitudinales y procedimentales; buscando profundizar los escenarios de reflexión, análisis e interpretación de los cambios globales que están sucediendo en los tiempos actuales. Por consiguiente, se está formando en las capacidades y habilidades para la mediación de los conflictos ambientales.

En América Latina, la educación para el desarrollo sustentable se centra en el aspecto académico, sin embargo, se ha observado ciertas dificultades por los especialistas y entes sociales. Ellos visionan una postura que incide en el campo de la educación ambiental. También, han efectuado profundas críticas a este tipo de educación que han sido promulgadas por entes internacionales y en el transcurrir del tiempo han recatado el término de sustentabilidad.

En este orden de ideas, en Colombia, según el Plan Nacional de Seguridad Alimentaria y Nutricional (PNSAN, 2012-2019): expone que "hace más de tres décadas comenzó a diseñar y aplicar estrategias de alimentación y nutrición, con un enfoque de subsidios y ayuda humanitaria otorgados a través de diferentes instancias con el propósito de favorecer a las familias más pobres" (pág. 11). Esto refiere que un país como Colombia gestiona, desde hacía varias décadas, el beneficio alimenticio para las comunidades menos vulnerable, con la finalidad de implementar herramientas comunitarias en función de fortalecer el bienestar y vida de los individuos.

En este sentido, en los países como Bolivia y Ecuador, enmarcan las 
Políticas de Seguridad Alimentaria en una soberanía alimentaria, según la Comunidad Andina (2010): señala que "está sustenta en cuatro pilares: el derecho a la alimentación; el acceso a los recursos productivos; la promoción de una producción agroecológica; la promoción y protección de los mercados locales y el cambio de las reglas del mercado internacional" (pág. 7). Es decir, que la seguridad alimentaria en Bolivia y Ecuador están basadas en el derecho que tienen los ciudadanos a la alimentación a través de la producción de productos; así como la protección y el resguardo de estos.

Lo antes expuesto conduce a verificar que en otros países se ha venido resguardando la seguridad alimentaria por medio de la producción de diferentes rubros en beneficio y seguridad de los habitantes. Tal caso ocurre en Venezuela con el proyecto de patios productivos. El mismo surge como una necesidad de sectores afectados por índices de pobreza y es a partir del gobierno del presidente Hugo de los Reyes Chávez se incorpora como política de Estado.

Igualmente, el presidente Hugo de los Reyes Chávez, comandante supremo ya fallecido, designa a través del Instituto Nacional de Investigaciones Agrícolas (INIA), ente adscrito al Ministerio del Poder Popular para la Agricultura Productiva y Tierras el trabajo de la agricultura familiar que consiste en la producción de varios rubros y diferentes cultivos en función de elevar el bienestar social, económico y educativo de los ciudadanos y ciudadanas de todo el territorio de Venezuela.

En este orden de ideas, los patios productivos como estrategia requieren el impulso de valores que incentiven los modelos de consumo humano en función de mantener, ecológicamente, el ambiente. Los patios vienen a incorporarse como una estrategia de desarrollo en función de ubicar esfuerzos mancomunados en el fortalecimiento de la participación y cooperación entre el Estado y la sociedad, asimismo la incorporación de la comunidad científica, las empresas privadas en conjunto con los entes 
gubernamentales y la comunidad en general.

Partiendo de lo antes expuesto, se tiene que en la Escuela Básica "Francisco de Rivas", ubicada en la urbanización Andrés Bello del municipio Barinas, estado Barinas, a través de la observación directa, los docentes tienen desconocimiento en la implementación de patios productivos como estrategia; razón por la cual ofrecen resistencia a los cambios y transformaciones a las políticas de Estado; haciendo a un lado la importancia que tienen los mismos en la economía familiar.

Por lo tanto, la ausencia de patios productivos como estrategia en la consolidación de la seducción ambiental trae como causas: incumplimiento de las políticas de Estado, desconocimiento de la relevancia de la implementación de patios productivos, falta de conocimiento de la importancia de la educación ambiental en la formación de valores en los educandos, desactualización del docente en la producción agroalimentaria y los beneficios para el colectivo estudiantil-comunitario y poca conservación del ambiente. Esto trae como consecuencias: ausentismo del docente a las funciones inherentes a su rol de promotor social, falta de integración de los estudiantes, padres y representantes a la producción alimentaria, aumento de la contaminación ambiental en la institución educativa, ausencia de interrelaciones con el ambiente y el entorno escolar.

De allí, surge la inquietud de realizar un estudio que tiene como objetivo proponer los patios productivos como estrategia para la consolidación de la educación ambiental en los docentes de la Escuela Básica "Francisco Rivas", ubicada en el municipio Barinas, estado Barinas. En vista que impulsará la producción de alimenticios impulsando hábitos en la alimentación y por ende la conservación del ambiente.

No obstante, la investigación generó las siguientes interrogantes: ¿Cuál es la situación existente en cuanto al uso de los patios productivos como estrategia para la consolidación de la educación ambiental por los docentes 
de la Escuela Básica "Francisco Rivas", ubicada en el municipio Barinas, estado Barinas?, ¿Cómo será el diseño de la propuesta de patios productivos como estrategia para la consolidación de la educación ambiental a los docentes de la Escuela Básica "Francisco Rivas", ubicada en el municipio Barinas, estado Barinas?, ¿Cuál es la factibilidad de la propuesta Proponer patios productivos como estrategia para la consolidación de la educación ambiental desde lo social, educativo, económico y ambiental? De esto se generó el objetivo general relacionado con proponer patios productivos como estrategia para la consolidación de la educación ambiental en los docentes de la Escuela Básica "Francisco Rivas", ubicada en el municipio Barinas, estado Barinas.

\section{Antecedentes, Teoría y Conceptos}

\subsection{Antecedentes}

Los antecedentes de la investigación son más que los estudios previos o realizados que corresponden a trabajos de ascensos, informes científicos relacionados con el problema, es decir, estudios efectuados con posterioridad a ésta y que guardan alguna vinculación con el problema en estudio. Zarraga (2014a): en el estudio titulado "Lineamientos de Supervisión para la Implementación del Programa Todos Manos a la Siembra en Educación Media General" (pág. 1). Se planteó como objetivo proponer lineamientos de supervisión para la implementación del programa todos vamos a la siembra en Educación Media General del Liceo Nacional Virginia Gil de Hermoso, extensión Los Cortijos de Lourdes, parroquia Mene de Mauroa, municipio Mauroa del estado Falcón.

La metodología estuvo centrada en las investigaciones proyectivas, con diseño no experimental, de campo y transeccional. Los sujetos involucrados fueron cuatro (04) docentes que dictan la asignatura Agricultura del primer año y tres (03) directivos, considerándose una población censal. La recolección de 
los datos se obtuvo a través de dos cuestionarios, el primero denominado Lineamientos de Supervisión y el segundo Programa Todos Manos a la Siembra, dirigido tanto a directivos como a docentes.

La autora concluyó que las líneas estratégicas se han fundamentado para favorecer el programa, el cual se visualiza una integración comunitaria, en la cual todas las instituciones trabajen conjuntamente, evidenciándose el esfuerzo creativo, interno, voluntario y planificado a partir de sus propios recursos humanos y materiales con la finalidad de indemnizar las necesidades alimentarias de los individuos.

El antecedente contribuye con elementos teóricos relacionado con los patios productivos, a pesar de que el trabajo hace referencia al Programa Todos Manos a la Siembre, el autor hace un apartado a la importancia de los espacios alternativos o patios productivos. Además, plantea las características más relevantes de la producción familiar y su inserción en las escuelas, la incorporación activa de los docentes, padres, madres, representantes y estudiantes.

\subsection{Patios Productivos como Estrategia}

Los patios productivos son espacios que se encuentran en el pleno centro de los hogares, es decir, lugares que se encuentran desocupados 0 vacíos aptos para cultivar cualquier rubro. Dichos patios pueden cultivarse vegetales, frutas, hortalizas que no requieren de condiciones climáticas para su desarrollo, crecimiento y suelos especiales que ameritan de fertilizantes especializados para su producción. Al respecto, Díaz (2013), plantea que los patios productivos:

Son procedimientos o sistemas compuestos de producción que son desarrollados en terrenos especiales o localizados en espacios cercanos a una comunidad o parcelas cercanas localidades organizadas. Los mismos son una vía directa y económica en el abastecimiento de los hogares y en las 
poblaciones con suficientes alimentos. Por lo tanto, el uso de los patios productivos garantiza la seguridad alimentaria de todos y por ende el respaldo a los productores agrícolas. También, la utilidad de los patios depende de las dimensiones alta productividad que pueden proporcionar diversas provisiones, viene sea de origen animal y vegetal por largo tiempo (pág. 56).

Interpretando al autor, los patios productivos representan una alternativa de producción a los miembros de una determinada comunidad o escuela; se enmarca en un medio inmediato y económico que busca satisfacer las necesidades económicas de las familias. La utilidad va a depender de las dimensiones del mismo; contribuyendo en la proporción de alimentos de producto vegetal o animal.

En este sentido, Mindiola, citado por Zarraga (2014b): plantea que los patios productivos "se fundamentan en promover la articulación intrainstitucional e interinstitucional, a través de un enfoque con contenidos curriculares agroecológicos que contribuyan a la formación integral de las comunidades educativas y del poder popular para garantizar la seguridad y soberanía alimentaria" (pág. 45). Esto refiere que los patios productivos tienen como finalidad la coyuntura responsable de cada uno de los autores educativos que hacen vida en la institución escolar con los actores de la comunidad en general en beneficio del colectivo.

\subsubsection{Importancia de los Patios Productivos}

La importancia de los patios productivos obedece a los beneficios que trae la soberanía alimentaria, es decir, la libertad que tienen todos los ciudadanos de sembrar cualquier rubro en un determinado espacio, bien sea en la escuela o la comunidad; garantizando el abastecimiento de alimentos nutritivos y cosechados en terrenos aptos para los mismos. Dicha importancia incide en el comportamiento de los individuos en alcanzar un bienestar y 
calidad de vida óptima.

Por otra parte, Cárdenas (2015): plantea que la importancia de los patios productivos "son espacios que son preparados por miembros de la escuela o comunidad, en la cual se aprovechan los espacios que no son utilizados" (pág. 23). Esto refiere que son lugares que se pueden encontrar en el contexto escolar o en una comunidad determinada; dichos lugares no son utilizados y sirven para la siembre de yuca, frijol, caraotas, garbanzos, plátanos, topochos, cría de gallinas, patos, chivos, entre otros rubros que son usados para la alimentación humana.

\subsubsection{Propósitos de los Patios Productivos}

Los patios productivos son espacios destinados a la producción, se traduce en sinónimo de trabajo e integración de los saberes comunitarios en la escuela y es una vía para integrar las áreas del conocimiento desde una perspectiva ambientalista para el logro del equilibrio hombre-naturaleza, con la práctica de una agricultura sin pesticidas y venenos, así como la incorporación al ambiente educativo un ritmo más natural, pausado, relajado, alegre y solidario. Al respecto, Pulido (2015), señala que los propósitos están enmarcados:

Se basa en tecnologías y métodos naturales, sencillos y económicos donde se aprovecha toda la materia orgánica que desechamos. *Fomenta la preservación de los ecosistemas al evitar la degradación y el agotamiento de los suelos. *En el terreno se puede sembrar todo el año y se diversifica la producción de hortalizas orgánicas cultivadas de manera natural en el suelo, lo cual le brinda alimentos a los grupos familiares y a la comunidad educativa (pág. 8).

Interpretando al autor, los patios productivos están signados como un excelente recurso en la consolidación de la Educación Ambiental porque accede que los educandos ser garantes de su propio aprendizaje, 
enriqueciendo el conocimiento, cooperación, solidaridad y trabajo en equipo, la cooperación, la participación y adoptar diversos puntos de vistas críticos frente a lo que hacen, cumpliendo al mismo tiempo con uno de los propósitos de la educación ambiental, como lo es el de la participación en todas las actividades relacionadas con la agricultura.

\subsubsection{Seguridad Alimentaria}

La seguridad alimentaria surge como una proyección preventiva. La misma tiene como propósito garantizar el acceso de los alimentos básicos a una determinada comunidad, incorporando la capacidad económica de obtenerlo, los suministros y los recursos que pueden obtener ha mediado 0 largo plazo. Al respecto, Jácome y Falcones (2012), plantea:

La Seguridad Alimentaria se mide según la capacidad de la familia para garantizar suficientes alimentos que permitan satisfacer todas las necesidades de nutrientes; los requerimientos de los micronutrientes esenciales y los requerimientos energéticos de todos los miembros de la familia. Debe priorizar las necesidades de los niños, los jóvenes y las mujeres en edad fértil (pág. 2).

Los autores refieren, la seguridad alimentaria comprende la capacidad que tienen las familias responder por la cantidad de alimentos que acceden a proporcionar todas las necesidades de sustento, obligaciones y aspectos enérgicos que abarque a todos los integrantes de los hogares; prevaleciendo a los niños, niñas, adolescentes, adultos y adultos mayores.

\subsection{Consolidación de la Educación Ambiental}

La educación se centra en una de las prácticas más antiguas y relevantes en la transformación social, histórica y cultural que ha acontecido en los últimos tiempos. Mendoza (2017): indica que la educación ambiental "es fundamental para la formación de los ciudadanos con el fin de construir una 
cultura ecológica que mejore la relación del hombre con el ambiente" (pág. 318). Es decir, representa un aspecto primordial en la preparación de la conciencia social y en la sistematización científica en la construcción de saberes de lo cultural y social.

Por consiguiente, Gabaldón, citado por Requena (2018): expone que la educación ambiental representa "la identificación de los problemas ambientales y cómo controlarlos requiere un nuevo tipo de educación que a través de un juicio teórico y práctico del medio biótico y físico cree el interés y el amor por la naturaleza" (pág. 296). El autor refleja, la educación ambiental incide en la formación de los individuos de manera teórico y práctico en función de mejorar el ambiente.

\subsubsection{Problemas Ambientales}

Los problemas ambientales están relacionados con las adversidades o revueltas que son generadas en el contexto ambiental. Es así como puede tratarse de los efectos de la contaminación, con derrames de petróleo que son vertidos en los océanos o los gases que son liberados a la atmósfera. González (2012): expone que los problemas ambientales "hacen referencia a una actitud de carácter general hacia el ambiente 0 al conjunto de orientaciones más o menos específicas que los seres humanos al mantenerse hacia los diferentes temas o sucesos ambientales" (pág. 56).

El autor, antes mencionado, refleja la importancia que tiene de mantener una actitud hacia la preservación del ambiente. Las mismas deben provenir de los individuos en mantener la calidad de vida social. Igualmente, fomentar el dominio de las cualidades ambientales que tienen sobre el comportamiento con respecto a diversos aspectos relacionados con el ambiente, hasta el punto de predecir o manifestar la manera suficiente de la conducta hacia el ambiente. De igual manera, García y Rosales (2012), exponen que: 
Los problemas ambientales con tantas implicaciones, para poder analizarlos, es necesario abordarlos parcialmente y al resolver cada una de las partes se llegaría a una solución global, asimismo se podrá abordar la problemática ambiental empleando enfoques o vertientes como: lo político-económico, lo técnico y lo socio educativo (pág. 34).

De allí que, los problemas ambientales son muy complejos de analizarlos, sin embargo, se pueden abordar ejecutando diversos enfoques socioeducativos, económicos, políticos y culturales que permitan generar una solución holística a dichos problemas; para ello se requiere de un proceso de socialización, sensibilización y cambios de actitud en el comportamiento de cada uno de los individuos o colectivos que hacen vida en las comunidades, escuelas y demás contextos involucrados.

\section{Metodología}

La investigación objeto de estudio se enmarcó en una investigación cuantitativa, razón por la cual acede a inspeccionar los datos científicamente de manera numérica, especialmente con la asistencia de las herramientas del campo de la estadística, utilizando datos agrupados con frecuencia absoluta y porcentajes. Monje (2011): indica que para que exista metodología cuantitativa "se debe buscar explicación a los fenómenos estableciendo regularidades en los mismos, esto es, hallar leyes generales que explican el comportamiento social" (pág. 11). En otras palabras, que exista claridad en cada uno de los aspectos indagados en el estudio que integran el problema, en la cual se defina la problemática, limitarlo y verificar puntualmente donde inicia, cual es el enfoque y la incidencia entre sus componentes.

En este orden y dirección, la investigación se focalizó en la modalidad de Proyecto Factible; debido a que el objetivo está centrado en proponer patios productivos como estrategia para la consolidación de la educación ambiental a los docentes de la Escuela Básica "Francisco Rivas", ubicada en el municipio 
Barinas, estado Barinas. De acuerdo con lo anterior, Villafañe (2013): señala que el proyecto factible consiste en "...la preparación y ejecución de acciones estratégicas como un modelo operativo orientado hacia la solución de un problema" (pág. 67). Esto refiere, proyecto factible consiste en un conjunto de acciones que van a solventar o mediar situaciones problemáticas en la realidad objeto de estudio; permitiendo solucionar de manera significativa el problema planteado mediante las diferentes fases propuestas en su metodología. Todo esto accederá a mejorar o consolidar contextos relacionado con la implementación de patios productivos.

En esta perspectiva, para llevar a cabo el proyecto factible, en primer lugar, se debe realizar un diagnóstico de la situación planteada; en segundo lugar, se procede a planear y fundamentar con basamentos teóricos la propuesta a diseñar y como se va a establecer tanto los procedimientos metodológicos, así como las actividades y los recursos necesarios para llevar adelante la ejecución de la misma. Igualmente, se efectuó el estudio de factibilidad del proyecto. Las fases o etapas por cumplir en la propuesta de investigación son: el diagnóstico, la factibilidad y el diseño de la propuesta.

Por su parte, el diseño del estudio se enmarcó en una investigación de campo, al respecto Rangel (2012): señala que “...una investigación es de campo, porque el investigador debe realizar diversas operaciones para obtener la información directamente de la realidad, sin ningún tipo de intermediación" (pág. 62). El conocimiento a profundidad del problema investigado y así manejar los datos obtenidos con mayor seguridad.

De allí que, la población constituye el universo de personas con las que se desarrolla la investigación. En tal sentido, dicha población estuvo determinada por el conjunto de personas que conformaron el contexto del estudio con sus características definitorias, atendiendo a los docentes de la Escuela Básica "Francisco Rivas", conformada por sesenta y cinco (65) profesionales de la docencia; tomando la totalidad para la muestra objeto de 
estudio.

Las técnicas de obtención de datos, se define como aquellas formas de observar el contexto objeto de estudio, son procesos científicos, en otras palabras, se emplean la diversidad de herramientas con que cuenta el investigador para observar las diferentes situaciones presentadas y en esa medida conseguir la información que requiere para el desarrollo de la investigación. Por ello, se diseñó un cuestionario tipo encuesta bajo la escala de Likert, con cinco alternativas de respuesta: Siempre (S), Casi Siempre, Algunas Veces (AV), Casi Nunca (CN) y Nunca (N).

\section{Resultados}

En este apartado se exponen los resultados de la investigación con sus respectivos análisis e interpretación, obtenidos con la aplicación del instrumento de recolección de datos y el uso de un cuestionario con escalamiento tipo Likert, con las alternativas: Siempre, Casi Siempre, Algunas Veces, Casi Nunca y Nunca tomados de acuerdo con los datos aportados por treinta y cinco (35) docentes de la Escuela Básica "Francisco Rivas". El mismo representa la primera fase del proyecto factible, a saber, el diagnostico. A continuación, se ilustran algunos resultados.

Cuadro 1. Distribución de la Frecuencia de la Opinión de los Docente con la Variable Patios Productivos como Estrategia, Dimensión Educativa e indicadores Importancia y Propósito.

\begin{tabular}{|c|c|c|c|c|c|c|c|c|c|c|c|}
\hline N.․ & Ítems & \multicolumn{2}{|c|}{ Siempre } & \multicolumn{2}{|c|}{$\begin{array}{c}\text { Casi } \\
\text { Siempre }\end{array}$} & \multicolumn{2}{|c|}{$\begin{array}{c}\text { Algunas } \\
\text { Veces }\end{array}$} & \multicolumn{2}{|c|}{ Casi Nunca } & \multicolumn{2}{|c|}{ Nunca } \\
\hline \multirow[b]{2}{*}{1} & \multirow{2}{*}{$\begin{array}{l}\text { Considera que la importancia } \\
\text { de los patios productivos } \\
\text { obedece a los beneficios que } \\
\text { trae la soberanía alimentaria. }\end{array}$} & $\mathrm{Fa}$ & $\%$ & $\mathrm{Fa}$ & $\%$ & $\mathbf{F a}$ & $\%$ & $\mathrm{Fa}$ & $\%$ & $\mathbf{F a}$ & $\%$ \\
\hline & & 7 & 11 & 18 & 28 & 21 & 32 & 5 & 8 & 14 & 21 \\
\hline
\end{tabular}

Fuente: Variable Patios Productivos; La Autora (2017) 
Cuadro 1. (Continuación). Distribución de la Frecuencia de la Opinión de los Docente con la Variable Patios Productivos como Estrategia, Dimensión Educativa e indicadores Importancia y Propósito.

\begin{tabular}{|c|c|c|c|c|c|c|c|c|c|c|c|}
\hline NN.으 & Ítems & \multicolumn{2}{|c|}{ Siempre } & \multicolumn{2}{|c|}{$\begin{array}{c}\text { Casi } \\
\text { Siempre }\end{array}$} & \multicolumn{2}{|c|}{$\begin{array}{l}\text { Algunas } \\
\text { Veces }\end{array}$} & \multicolumn{2}{|c|}{ Casi Nunca } & \multicolumn{2}{|c|}{ Nunca } \\
\hline & $\begin{array}{l}\text { Uno de los propósitos } \\
\text { de los patios }\end{array}$ & $\mathrm{Fa}$ & $\%$ & $\mathrm{Fa}$ & $\%$ & $\mathrm{Fa}$ & $\%$ & $\mathrm{Fa}$ & $\%$ & $\mathrm{Fa}$ & $\%$ \\
\hline 2 & $\begin{array}{c}\text { productivos consiste } \\
\text { en la promoción de } \\
\text { formas de vida más } \\
\text { armoniosas, } \\
\text { equilibradas con la } \\
\text { naturaleza y los seres } \\
\text { vivos al fomentar la } \\
\text { participación entre } \\
\text { ellos. }\end{array}$ & 8 & 12 & 16 & 25 & 24 & 37 & 5 & 8 & 12 & 18 \\
\hline
\end{tabular}

Fuente: Variable Patios Productivos; La Autora (2017).

En el cuadro 1, el ítem el 1 el $32 \%$ de los docentes encuestados indicaron Algunas Veces consideran que la importancia de los patios productivos obedece a los beneficios que trae la soberanía alimentaria, el 28\% Casi Siempre, el 21\% Nunca, el 11\% Siempre y el 9\% Casi Nunca. El ítem 2, el $37 \%$ señalaron Algunas Veces uno de los propósitos de los patios productivos consiste en la promoción de formas de vida más armoniosas, equilibradas con la naturaleza y los seres vivos al fomentar la participación entre ellos, el $25 \%$ Casi Siempre, el 18\% Nunca, el 12\% Siempre y el $8 \%$ Casi Nunca.

Los datos obtenidos infieren, los educadores consideran que medianamente la importancia de los patios productivos obedece a los beneficios que trae la soberanía alimentaria; así como también con poca frecuencia uno de los propósitos de los patios productivos consiste en la promoción de formas de vida más armoniosas, equilibradas con la naturaleza y los seres vivos al fomentar la participación entre ellos. 
Cuadro 2. Distribución de la Frecuencia de la Opinión de los Docente con la Variable, Consolidación de la Educación Ambiental, Dimensión Ámbito Social e indicadores Educación Ambiental y Características.

\begin{tabular}{|c|c|c|c|c|c|c|c|c|c|c|c|}
\hline N.ㅇ & Ítems & Sie & & $\begin{array}{r}\mathrm{C} \\
\mathrm{Sie}\end{array}$ & & $\begin{array}{r}\text { Alg } \\
\text { Ve }\end{array}$ & & & & $\mathrm{Nu}$ & \\
\hline \multirow[b]{2}{*}{3} & \multirow{2}{*}{$\begin{array}{l}\text { Utiliza la educación } \\
\text { ambiental en la } \\
\text { consolidación de } \\
\text { patios productivos } \\
\text { en la escuela. }\end{array}$} & $\mathrm{Fa}$ & $\%$ & $\mathrm{Fa}$ & $\%$ & $\mathbf{F a}$ & $\%$ & $\mathrm{Fa}$ & $\%$ & $\mathrm{Fa}$ & $\%$ \\
\hline & & 0 & 0 & 0 & 0 & 16 & 25 & 9 & 14 & 40 & 61 \\
\hline 4 & $\begin{array}{c}\text { Considera que una } \\
\text { de las } \\
\text { características de la } \\
\text { educación ambiental } \\
\text { está centrada en la } \\
\text { sostenibilidad. }\end{array}$ & 0 & 0 & 0 & 0 & 36 & 55 & 7 & 11 & 22 & 34 \\
\hline
\end{tabular}

Fuente: Variable Patios Productivos; La Autora (2017).

En el cuadro 2, en el ítem 3 el 61\% de los encuestados respondieron Nunca utilizan la educación ambiental en la consolidación de patios productivos en la escuela, el 25\% Algunas Veces y el 14\% Casi Nunca. El ítem 4 , el $55 \%$ de los educadores considera que Algunas Veces una de las características de la educación ambiental está centrada en la sostenibilidad, el $34 \%$ Nunca y el $11 \%$ Casi Nunca.

Los resultados obtenidos inducen a analizar que la gran mayoría de los docentes carecen de la utilización de la educación ambiental en la consolidación de patios productivos. Igualmente, con poca frecuencia una de las características de la educación ambiental está centrada en la sostenibilidad.

\section{Propuesta}

Los patios productivos como estrategia vienen a constituir en las instituciones educativas una alternativa de espacios para la producción de diferentes rubros, entre ellos animal, vegetal y frutal con la finalidad de 
consolidar la educación ambiental en el plantel. Además, la producción de ellos genera beneficios a la colectividad estudiantil y por ende ingresos económicos para la gestión administrativa y académica del plantel.

\subsection{Objetivos de la Propuesta}

\subsubsection{Objetivo General:}

Diseñar la propuesta de patios productivos como estrategia para la consolidación de la educación ambiental a los docentes de la Escuela Básica "Francisco Rivas", ubicada en el municipio Barinas, estado Barinas.

\subsubsection{Objetivos Específicos}

1. Sensibilizar a los docentes para que participen en la propuesta de patios productivos como estrategia para la consolidación de la educación ambiental.

2. Promover colectivos de formación relacionado con los patios productivos, importancia, relevancia social, económica y educativa.

3. Capacitar en la educación ambiental, seguridad alimentaria, desarrollo endógeno y desarrollo sustentable a los docentes de la escuela.

4. Propiciar diálogos de saberes con los docentes con la finalidad de fortalecer los patios productivos en la escuela y la comunidad.

5. Promover la difusión y divulgación de los patios productivos como estrategia y la consolidación de la educación ambiental en los estudiantes de Educación Primaria.

\subsection{Contenido del Plan de Acción}

El contenido de plan acción refleja los objetivos específicos, la estrategia, actividades, tiempo, responsable y recursos con el propósito de establecer claramente cada una de las acciones que se seguirán para su posterior ejecución. Además, se exponen cada objetivo específico de la 
propuesta con sus respectivas actividades y lapso de ejecución con el propósito de tener una visión y sistematización en el proceso de sensibilización, capacitación, formación, compartir de saberes y promoción de los patios productivos en consolidación de la educación ambiental.

Cuadro 3. Planificación del objetivo específico 1, relacionado con el plan de acción de patios productivos como estrategia para la consolidación de la educación ambiental.

\begin{tabular}{|c|c|c|c|c|}
\hline \multicolumn{5}{|c|}{$\begin{array}{l}\text { Objetivo Específico 1: Sensibilizar a los docentes para que participen en la propuesta } \\
\text { de patios productivos como estrategia para la consolidación de la educación ambiental. }\end{array}$} \\
\hline Estrategia & Actividades & Tiempo & Responsables & Recursos \\
\hline $\begin{array}{l}\text { Se efectuará } \\
\text { por medio del } \\
\text { proceso de } \\
\text { sensibilización }\end{array}$ & $\begin{array}{l}\text { - Conversación con el } \\
\text { equipo directivo con } \\
\text { la finalidad de solicitar } \\
\text { permiso para el } \\
\text { desarrollo de la } \\
\text { propuesta de patios } \\
\text { productivos como } \\
\text { estrategia para la } \\
\text { consolidación de la } \\
\text { educación ambiental } \\
\text { - Convocatoria, por } \\
\text { medio de volantes, a } \\
\text { los docentes a la } \\
\text { jornada de } \\
\text { presentación del plan. } \\
\text { - Realización de la } \\
\text { jornada con dinámica } \\
\text { de presentación. } \\
\text { - Proyección del plan } \\
\text { de acción a los } \\
\text { asistentes empleando } \\
\text { el recurso video } \\
\text { Beam. }\end{array}$ & 2 semanas & Investigadora & $\begin{array}{l}\text { Humanos: } \\
\text { Directivos } \\
\text { Docentes } \\
\text { Investigador. } \\
\text { Materiales: } \\
\text { Fotocopias } \\
\text { Registro de } \\
\text { Asistencias. } \\
\text { Evaluación: } \\
\text { Formativa: } \\
\text { Registro de } \\
\text { Asistencias } \\
\text { Fotografías } \\
\text { Acta de reunión. } \\
\text { Criterio: } \\
\text { Responsabilidad } \\
\text { Participación } \\
\text { Sensibilización. } \\
\text { Indicadores: } \\
\text { Puntualidad a la } \\
\text { jornada } \\
\text { Manejo del } \\
\text { conocimiento. }\end{array}$ \\
\hline
\end{tabular}

Fuente: La Autora (2017).

\section{Conclusiones y Recomendaciones}

La propuesta fue diseñada y planificada por medio de un plan de acción contentivo de: objetivo específico, estrategias, actividades, recursos, responsable y evaluación. Por lo tanto, se generó la propuesta que posteriormente será aplicada por la comunidad objeto de estudio, otros entes, 
instituciones u organizaciones. Con la aplicación del diagnóstico se obtuvo los siguientes resultados:

Los educadores, medianamente consideran que la importancia de los patios productivos obedece a los beneficios que trae la soberanía alimentaria; así como también con poca frecuencia uno de los propósitos de los patios productivos consiste en la promoción de formas de vida más armoniosas, equilibradas con la naturaleza y los seres vivos al fomentar la participación entre ellos.

En cuanto al estudio de la factibilidad se focaliza la viabilidad de implementación de la misma; planteando que a nivel económico solo ocasionará gastos relacionados con el material y refrigerio que se va a utilizar cuando se desarrollen los colectivos de formación, charlas y jornadas, las mismas serán asumidas por la investigadora y la directiva del plantel.

\section{Referencias}

Cárdenas, B. (2015). Importancia de los Patios Productivos. Colombia: Trillas.

Comunidad Andina (2010). Estrategias, Políticas y Acciones de Seguridad

Alimentaria para Poblaciones Indígenas en los Países de la Comunidad Andina. Perú: Congreso de la República. Recuperado de: http://www2.congreso.gob.pe/sicr/cendocbib/con4 uibd.nst/9AA6BAD4 C9A669D305257EEA00628FBF/\$FILE/EstrategiasPol\%C3\%ADticas y AccionesSeguridadAlimentaria.pdf

Díaz, C. (2013). Patios Productivos. Colombia: Norma.

García, C., \& Rosales, V. (2012). Problemas Ambientales a Nivel Mundial. Buenos Aires, Argentina: Ares.

González, R. (2012). Problemas Ambientales en Venezuela. Caracas, Venezuela: Editores.

Jácome, R., \& Falcones, A. (2012). Medición de la Seguridad Alimentaria y 
Nutricional en Familias con Niños/As Menores de 5 Años de la Comunidad el Cerotal, Cantón Antonio Ante, Provincia de Imbabura. Ecuador: Universidad Técnica del Norte. Recuperado de: http://repositorio.utn.edu.ec/handle/123456789/2159

Mendoza, Y. (2017). Fortalecimiento de la Educación Ambiental a Través de la Siembra de Plantas Ornamentales. Revista Scientific, 3(7), 309328. Recuperado de: https://doi.org/10.29394/Scientific.issn.25422987.2018.3.7.16.309-328

Monje, C. (2011). Metodología de la Investigación Cuantitativa y Cualitativa. Guía Didáctica. Colombia: Universidad Surcolombiana. Recuperado de: https://www.uv.mx/rmipe/files/2017/02/Guia-didacticametodologia-de-la-investigacion.pdf

PNSAN (2012). Plan Nacional de Seguridad Alimentaria y Nutricional (PNSAN) 2012-2019. Colombia: Comisión Intersectorial de Seguridad Alimentaria y Nutricional. Gobierno Nacional de Colombia. Recuperado de: http://www.osancolombia.gov.co/doc/pnsan.pdf Pulido, J. (2015). Propósitos de los Patios Productivos. Colombia: Norma. Rangel, M. (2012). Metodología de la Investigación. Barinas, Venezuela: Ediciones de la Universidad Nacional Experimental de los Llanos Occidentales "Ezequiel Zamora".

Requena, Y. (2018). Investigación Acción Participativa y Educación Ambiental. Revista Scientific, 3(7), 289-308. Recuperado de:

https://doi.org/10.29394/Scientific.issn.2542-2987.2018.3.7.15.289-308 Villafañe, M. (2013). Metodología de la Investigación. Venezuela: Trillas. Zarraga, L. (2014a,b). Lineamientos de Supervisión para la Implementación del Programa "Todas las Manos a la Siembra" en Educación Media General. Trabajo de Grado de Maestría. Maracaibo, Venezuela: Universidad Rafael Urdaneta. Recuperado de: http://200.35.84.131/portal/bases/marc/texto/9217-14-08699.pdf 


\section{Bárbara Ysabel Díaz Vásquez \\ e-mail: marlenism3@gmail.com}

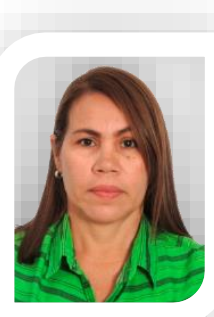

Nacida en el municipio Alberto Arvelo Torrealba, Sabaneta del estado Barinas, Venezuela. Licenciada en Educación Integral de la Universidad Nacional Experimental "Simón Rodríguez". Experiencia profesional, docente de aula fija en la Escuela Básica "José Francisco Jiménez", municipio Pedraza. Actualmente laboro como docente de aula en la Escuela "Francisco Rivas", municipio Barinas. En jornada de formación: Operador de Micro Bajo Ambiente Windows 98. Instituto Andrés Bello, Taller de Juegos y Canciones para el Aula. 